\title{
Italique
}

Poésie italienne de la Renaissance

XII | 2009

Varia

\section{A margine di Piansi et cantai del Bembo}

\section{Giovanni Ferroni}

\section{OpenEdition}

\section{Journals}

Édition électronique

URL : http://journals.openedition.org/italique/224

DOI : 10.4000/italique.224

ISSN : 1663-4438

\section{Éditeur}

Librairie Droz

\section{Édition imprimée}

Date de publication : 1 novembre 2009

Pagination : 73-91

ISSN : 1423-3983

\section{Référence électronique}

Giovanni Ferroni, «A margine di Piansi et cantai del Bembo », Italique [En ligne], XII | 2009, mis en ligne le 01 novembre 2012, consulté le 01 mai 2019. URL : http://journals.openedition.org/italique/224 ;

DOI : $10.4000 /$ italique.224

\section{(c) Tous droits réservés}


Giovanni FERroni

A MARGINE D I

PIANSI ET CANTAI

D E L B E M B O 



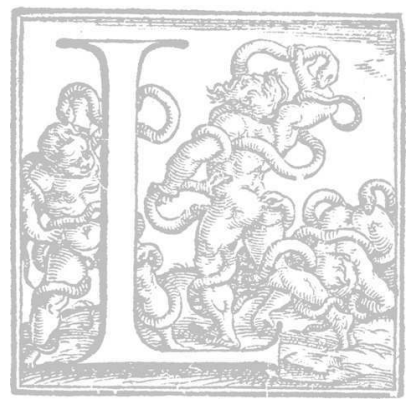

A carta che reca impresso il sonetto d'inizio delle Rime di Monsignor Pietro Bembo non presenta ormai molto spazio libero: nei secoli e più ancora in anni recenti essa si è via via riempita di fitte annotazioni filologiche, esegetiche e critiche, nelle possibili dimensioni, verticale e orizzontale, della lettura. I fondamenti di poetica che il testo presuppone, ${ }^{\mathrm{I}}$ le tessere petrarchesche intarsiate, la qualità e $i$ modi dell'imitatio, ${ }^{2}$ sono già stati individuati e illustrati in maniera esauriente, potendo così servire da riferimento al nostro discorso; già scritta nelle sue linee essenziali e solo bisognosa di pochi aggiornamenti è poi la storia della (scarsa) fortuna critica del testo, ${ }^{3}$ cosicché, dandola per nota e richiamandone all'occorrenza alcuni momenti, si potrà procedere, senz'altro intervallo, all'esposizione dei limitati rilievi che qui si propongono e che toccheranno principalmente l'organizzazione retorica del sonetto. Per comodità, ripropongo di seguito il pur notissimo testo: ${ }^{4}$

$$
\begin{aligned}
& \text { Piansi et cantai lo stratio et l'aspra guerra, } \\
& \text { ch'i' ebbi a sostener molti et molt'anni, } \\
& \text { et la cagion di così lunghi affanni, } \\
& \text { cose prima non mai vedute in terra. } \\
& \text { Dive, per cui s'apre Helicona et serra, } \\
& \text { use far a la morte illustri inganni, } \\
& \text { date a lo stil, che nacque de' miei danni, } \\
& \text { viver quand'io sarò spento e sotterra. } \\
& \text { Ché potranno talhor gli amanti accorti, } \\
& \text { queste rime leggendo, al van desio } \\
& \text { ritoglier l'alme col mio duro exempio; } \\
& \text { et quella strada, ch'a buon fine porti, } \\
& \text { scorger da l'altre, et quanto adorar Dio } \\
& \text { solo si dee nel mondo, ch'è suo tempio. }
\end{aligned}
$$

Cominciamo da una somiglianza che altri ha già notato: il verso conclusivo della prima quartina, "cose prima non mai vedute in terra ", suona singolarmente simile a quel « cosa non detta in prosa mai né in rima » che definisce, nel principio dell'opera, fin dall'edizione del IsIs, l'eccezionale originalità narrativa del Furioso. E questo un fatto, ben me ne rendo conto, trascurabile, ma la vicinanza tra due testi cosi diversi e cosi esemplari è, a suo modo, un piccolo scandalo che merita d'essere approfondito. Certo, come si sa, il verso del Bembo, contrariamente a quello dell' Ariosto, ne echeggia uno del Triumphus Cupidinis che lo autorizza; ${ }^{6}$ certo, al di là delle esatte coincidenze lemmatiche 
fra i due versi, l'assonanza fonica è un'acquisizione 'tarda', dovuta all'opera correttiva del Bembo che mutò l'originario "cose rado o non mai... $\rangle^{7}$ nel definitivo "cose prima... », introducendo un avverbio che, nella chimica dei fonemi, risulta, fra la sciarada e il calembour, dalla concentrazione delle ariostesche "prosa mai » e "rima »; certo, tutto ciò può essere, anz̧i lo sarà di sicuro, più che un'assonanza, una banale e involontaria coincidenza. Non si può tuttavia ignorare che tra $i$ due versi esiste un'affinità vera, radicata nella genesi dei testi, che si situa a monte di ogni processo correttorio e che è evidentemente posta non tanto sul piano dell'elocutio quanto su quello dell'inventio e del compito retorico attribuito agli enunciati: quel che entrambi rimarcano è l'assoluta novità delle opere, delle narrazioni che introducono.

Per far meglio risaltare questo dato è necessario restituirgli quella, minima, profondità di prospettiva teorica che gli spetta, cominciando cioè col richiamare una nozione scolastica: la parte, di cui stiamo trattando e a cui appartengono i versi del Bembo e dell'Ariosto, è l'exordium. Prima, nella trattażione della Rethorica ad Herennium, ${ }^{8}$ delle « sex partes orationis » di cui è composta l'inventio, esso è definito "principium orationi, per quod animus auditoris constituitur ad audiendum $\gg .9$ Ciò, come si sa, comporta l'adempimento di tre officia: rendere l'ascoltatore attentum, docilem, benivolum. Ora, secondo l'insegnamento di Cornificio, otterremo l'altrui attenzione "si pollicebimur nos de rebus magnis, novis, inusitatis verba facturos ». ${ }^{\circ}{ }^{E}$ evidentemente quanto procacciano di fare tanto Ariosto quanto Bembo; anzi la trafila correttoria di quest'ultimo sul quarto verso del suo sonetto - da « cose rado o non mai $[. .]$.$» a «cose prima non mai [. .]$.$» - sembra proprio indicare un$ progresso nell'adesione ai precetti dei retori antichi. Eliminare ogni attenuazione infatti, significa puntare, come fin dall'inizio aveva fatto l'A riosto, su una richiesta d'attenzione fondata sul proclama quasi iperbolico - e parranno "esagerazioni idropiche » al Baretti ${ }^{1 \mathrm{I}}$ - della novitas e della magnitudo delle cose di cui si parlerà.

Non mi sembra che questo ricorso, da parte del Bembo, a uno dei luoghi retorici più ovvi sia mai stato segnalato, e forse perché fin troppo ovvio, perché fin troppo naturale doveva esserne l'impiego per scrittori che quelle regole avevano sulla punta delle dita. Ritengo tuttavia che un riferimento cosi esplicito agli insegnamenti della Rhetorica ad C. Herennium, o del De inventione che è lo stesso, in un testo del quale è inutile ripetere l'importanza, sia degno di rilievo e lo sia, come si vedrà, per più motivi.

E notevole intanto che il rispetto dei precetti relativi all'exordium non si limiti al solo quarto verso ma si estenda a tutto il sonetto, costituendo cosi la ragione teorica che presiede alla sua partitura argomentativa e, con questa, alla scelta di un ben definito progetto retorico. Riprendiamo dallinizio. La 
prima quartina del sonetto riassume la materia del canto, "lo strazio », "l'aspra guerra », l'estensione temporale, " la cagion", e, come già si è visto, cerca di attrarre l'attenzione dell'uditore. Il compito che le viene assegnato non $\grave{e}$ dunque indifferente, ma vale anzi ad inserire il testo, nel suo insieme e fin dalle sue prime battute, in uno degli schemi previsti dai trattatisti antichi. Recita infatti la Rhetorica ad C. Herennium: "Dociles auditores habere poterimus, si summam causae breviter exponemus et si attentos eos faciemus; nam docilis est qui attente vult audire ». ${ }^{\mathrm{I}}$ Cosi la prima quartina, con la succinta proposta della sua inusitata materia, serve al Bembo per acquistarsi la docilità e l'attenzione, in definitiva coincidenti, del suo pubblico. Ora, tanto Cornificio quanto Cicerone insegnavano a scegliere l'officium da compiere presso l'uditorio - renderlo attento, docile o benevolo - in relazione alla qualità della causa che l'oratore dovesse discutere. ${ }^{\mathrm{I}}$ Secondo tale dottrina, l'attenzione del pubblico si sarebbe dovuta ricercare nel caso in cui il genus causae fosse " humile, quod neglegitur ab auditore et non magno opere adtendendum videtur ». ${ }^{\mathrm{I}}{ }^{\mathrm{E}}$ allora proprio l'humilitas della causa, e cioè, se intendo bene, la scarsa rilevanza della materia amorosa, e quindi del genere elegiaco, che costringe il Bembo a occupare la prima quartina del sonetto nel modo che si è visto e soprattutto, per contrastare la temuta scarsa attenzione dell'uditorio, a proclamare l'inaudita novità delle vicende per le quali ha pianto e cantato.

Con queste premesse si giunge alla vexata quaestio della seconda quartina, separata dalla precedente da un vistoso scarto argomentativo - il « salto [...] smisurato » del Baretti ${ }^{15}$ - e dedicata a un'invocazione alle Muse che, fino a quel momento, era senza precedenti nella storia della lirica volgare. Imitata dal Della Casa, criticata dal Castelvetro ma difesa dal Tasso, essa può essere spiegata con la volontà bembiana d'innalzare il tono del canto, diventando cosi l'emblema, come ha mostrato Noyer-Weidner, ${ }^{16}$ di quella istanza 'epica' che anima il testo. Osservata però dal punto di vista dell'argomentazione e del suo procedere, la seconda quartina assume anche un'altra, importantissima, funzione, quella cioè di rendere esplicito l'interlocutore del poeta con una mossa allocutiva dalla quale dipende quanto segue del testo. Sebbene infatti le «Dive » siano persone simboliche - da intendersi come la Poesia se non, col Tasso, come le "Intelligenze ${ }^{17}$ - esse paiono tuttavia ragionevolmente chiamate in causa dal Bembo poiché egli appare in questo testo nella veste del poeta che presenta la propria opera - com'è evidente fin dalla preponderante accezione letteraria di quel "piansi e cantai », che definisce modalità di poesia e di stile valide per tutta la raccolta - e si rivolge perciò a chi ha autorità sui versi e sulla loro durata. Questa appropriatezza nell'attribuzione dei ruoli di parlante, messaggio e ascoltatore, e dunque nella costruzione di un contesto poetico-discorsivo, ha però alla base un preciso scopo retorico. Le Muse infatti 
prendono il posto dei patres conscripti delle orazioni antiche e impersonano cosi $i$ giudici della causa presentata nel sonetto e resa tangibile dal 'corpo del reato': il libro ormai compiuto delle Rime. Ė alle Muse che il poeta si presenta e ad esse, dopo averne ottenuta la docilità e l'attenzione, domanda non altro che benevolenza.

In questa richiesta sta d'altr'onde una differenza notevole rispetto all'invocazione dell'epica tante volte accostata ai versi del Bembo dagli antichi e dai moderni commentatori poiché, mentre in quella il poeta chiede direttamente il canto e il racconto alla divinità, più o meno simbolica, ch'egli invoca, qui, dove solo il poeta è l'autore dell'opera, si chiede che essa gli sopraviviva per i fini didattici resi poi espliciti nelle terzine. Ora se è vero, come s'è detto, che l'appello alle Muse era senza esempio nella lirica volgare prima del Bembo, non mi pare tuttavia accettabile senza riserve l'affermazione di Erspamer, secondo il quale questa invocazione è un "ingrediente estraneo all' ambito lirico, mutuato dal genus sublime, dalla poesia epica», ${ }^{18}$ e neppure quella di Donnini che a quell'ambito gindica del tutto estranea anche "la richiesta dell'immortalità dei versi [...], che è funzionale all'istruzione continua dei lettori destinatari ${ }^{19}{ }^{19}$ Più e meglio che all'epica infatti, questa quartina bembiana può essere affiancata, come d'altr'onde fa lo stesso Donnini nel suo commento, ${ }^{20}$ a un passo della quindicesima elegia del primo libro degli Amores ovidiani:

Ergo, cum silices, cum dens patientis aratri depereant aevo, carmina morte carent. cedant carminibus reges regumque triumphi, cedat et auriferi ripa benigna Tagi!

vilia miretur vulgus; mihi flavus Apollo pocula Castalia plena ministret aqua, sustineamque coma metuentem frigora myrtum, atque a sollicito multus amante legar! pascitur in vivis Livor; post fata quiescit, cum suus ex merito quemque tuetur honos ergo etiam cum me supremus adederit ignis, vivam, parsque mei multa superstes erit. ${ }^{2 \mathrm{I}}$

Il rimando non vale certo né in modo puntuale per l'elocutio, né per la collocazione dell'elegia all'interno del liber, né per il contesto in cui il brano s'inserisce, ma può essere di qualche rilievo proprio per la connessione che vi è stabilita fra il tema dell' immortalità della poesia, dunque la perenne utilità dei versi per $i$ loro destinatari, e la sopravivivenza del poeta oltre la propria morte. D'altra parte questa connessione, d'ascendenza è vero epico-didattica, diviene, per la sede in cui si trova, elegiaca e, come tale, poteva essere accolta dal Bembo 
rientrando infatti nell'ambito generico in cui anche Piansi et cantai e la poesia che presenta, pur latamente, s'iscrivono. Semplificando: l'innovativa mescolanza d'elegia, eternità del canto e didassi era, in origine, ovidiana e di li forse il Bembo la riprese operando una scelta che, non di meno, segnava nella lirica volgare una netta discontinuità.

Se si torna poi, nello specifico, alla struttura argomentativa, ben si vede che ̀̀ nella costruzione del discorso, non tanto nella sostanza della preghiera, che il Bembo si ispira di nuovo al consiglio dei manuali di retorica: dei quattro modi per rendere benevoli gli ascoltatori, cioè $i$ giudici, egli adatta alle sue necessità quello " ab auditorum persona ${ }^{22}$ Essi, scrive Cicerone, saranno infatti ben disposti « si res ab iis fortiter, sapienter, mansuete gestae proferentur, ut ne qua assentatio nimia significetur, si de iis quam honesta existimatio quantaque eorum indicii et auctoritatis expectatio sit ostendetur $\gg{ }^{23}$ Eे soprattutto la prima parte di questo passo che interessa: nello specificare, elencandone le proprietà, a quali dive si rivolga, Bembo loda, indirettamente e senza sconfinare nell'adulazione, il potere dei suoi specialissimi giudici; un potere che negli «illustri inganni » con $i$ quali, tante volte nel passato (use far) ha vinto la morte, si dimostra ricco di sapientia, mansuetudo $e$ magnificentia. ${ }^{24}$ La domanda di benevolenza non è però, dal punto di vista del sistema retorico, priva di conseguenze poiché essa si aggiunge a quella di docilità e attenzione. Lo scarto argomentativo che è sempre stato notato, e cui si ̀̀ accennato sopra, la durezza del passaggio di strofa, dipendono in parte anche da questo. Nei termini formalizzati della disciplina retorica infatti, Bembo innesta sul genus causae humile il genus dubium o anceps. ${ }^{25} \dot{E}$ cosi definita quella causa che " habet in se [...] et honestatis et turpitudinis partem 》 e per la quale, nell'esordio, volendo neutralizzare quella parte disdicevole che potrebbe danneggiarci, "benivolentiam captare oportebit $» .{ }^{26}$ Accade cosi che mentre la presentazione di una materia non particolarmente elevata aveva avuto bisogno della richiesta d'attenzione e docilità, il dubbio circa l'eccellenza dello « stile » che, per esser nato dai « danni » amorosi, per l'appartenen za a uno stile elegiaco, ha in sé elementi d'imperfezione, spinge ad invocare le Muse e alla ricerca della benevolenza dei giudici. La duplicità degli oggetti del discorso - la materia del canto e lo stile - e la corrispondente duplicità dei fini dell'esordio sono il segno d'un progetto retorico non semplice e che cerca di tenere assieme istanze almeno in parte diverse.

Nelle terzine, che formano un blocco unico dal punto di vista dell'argomentazione, la non totale linearità del discorso bembiano diviene ancor più sottile poiché alle duplicità menzionate, si aggiunge quella dell'interlocutore. Se è infatti evidente che, anche qui, il destinatario 'reale', ma in verità fittizio, cui il poeta si rivolge sono le Dive, è facile vedere come il testo ne faccia intravedere un altro, implicito, ideale e non di meno più concreto del primo, vale a dire la 
schiera degli amanti futuri, dei potenziali lettori del libro. Al raddoppiarsi degli interlocutori corrisponde poi lo sdoppiarsi della figura dell'autore che non è più solo poeta - e anche, quando lo era, amante - ma anche maestro, e che assume cosi ruoli che richiedono differenti finalità retoriche e che ripropongono la duplicità degli oggetti del discorso e dei genera causarum.

Da un punto di vista sintattico predominano senz'altro il riferimento alle Muse, il ruolo di poeta, il genus dubium $e$ con esso la richiesta di benevolenza. Quel «Ché» infatti, con il quale si apre il nono verso del sonetto e che introduce $i$ motivi per $i$ quali è auspicabile la sopravvivenza dei versi oltre la vita del loro autore, introduce anche l'accenno a parte della topica " ab nostra persona» con cui si rendono benevoli gli ascoltatori. La menzione del « duro exempio » conservato nelle « rime » sembra cosi mettere in pratica il consiglio di ricordare "nostra incommoda [...] inopiam, solitudinem, calamitatem », mentre $i$ benefici che i lettori trarranno valgono a suscitare il favore nei confronti del poeta, poiché essi possono essere facilmente rubricati come l'ammaestramento derivante dal racconto di «in rem $p$. quales fuerimus aut in parentes aut in amicos aut in eos, qui audiunt $\gg .{ }^{27}$

D'altro canto, questi stessi versi sono funzionali anche al genus humile $e$ dunque alla necessità di richiamare l'attenzione. In essi infatti è la materia del libro e non più lo stile ad essere centrale, cosicché anche l'insegnamento che $i$ nuovi destinatari, gli « amanti accorti », traggono dall'esempio del maestro, può integrare, indirettamente, l'argumentum presentato nella prima quartina. A ciò si aggiunge il fatto che proprio $i$ capi nei quali si concretizzano gli insegnamenti contenuti nel libro, e cioè " al van desio / ritoglier l'alme», "quella strada, ch'a buon fine porti, / scorger» e "quanto adorar Dio / solo si dee », si avvicinano parecchio ai temi che Cornificio consigliava di promettere di discutere quando ci si volesse guadagnare l'interesse dell'uditorio, quelle cose cioè " quae ad rem p. pertineant, aut ad eos ipsos, qui audient, aut ad deorum inmortalium religionem $\gg{ }^{28}$

L'importanza dell'operazione bembiana, quanto cioè possa essere rilevante il riferimento diretto, seppur liberissimo, ${ }^{29}$ in un luogo eminente come l'esordio, ai precetti retorici del De inventione o della Rhetorica ad C. Herennium, diviene ancor meglio apprezzabile quando si riproponga un topos degli studi bembiani e, più in generale, degli studi sulla lirica del Cinquecento: il confronto di Piansi et cantai col testo incipitario del Canzoniere. Si tratta di un confronto interessante poiché, come è stato a più riprese notato, il sonetto del Bembo, pur essendo "quintessenzialmente petrarchesco $»^{3 \circ}$ nel metro e nelle voci, appare molto distante nei contenuti dal suo 'modello' trecentesco e questo non ostante l'esemplarità indiscutibile del testo di Petrarca. Di tale distanza e diversità si ha un riscontro anche conducendo un paragone fra le strutture retoriche dei due testi e particolarmente se si abbia l'avvertenza 
di avvicinare i due autori, di osservare cioè Petrarca attraverso il filtro di una delle sue mediazioni cinquecentesche.

Il commento al Canzoniere di Bernardino Daniello ${ }^{31}$ è senżaltro, per il comune riferimento al magistero di Trifon Gabriele, il più vicino al Bembo, alla sua cerchia e al modo che vi si teneva di leggere e interpretare il testo petrarchesco. La nota introduttiva del lucchese a Voi ch'ascoltate in rime sparse il suono è, per l'intendimento di questo contributo, illuminante e la riporto percio integralmente:

Dubitando il Poe[ta], d'esser piu tosto d'alcun biasimo, et infamia da coloro notato, alle mani, et alle orecchie de quali fossero per venire le cose sue; che d'honore et di lode riputato degno, come quegli che non solamente tutta la sua acerba et giovenile etate; ma buona parte della matura anchora dietro le vane et fallaci amorose cure, speso havea; fece il presente Sonetto, ultimo se non di tutti, almeno di quelli ch'esso volle che si leggessero, ponendolo primo in ordine di tutti gli altri Sonetti, et Canzoni, come Proemio fatto per insinuatione, preoccupando in esso quello c'havrebbon potuto dir gli ascoltanti, cioè non ben convenirsi ad huomo grave, et religioso, si com'egli era, il trattare et cantare di cose lievi, come sono queste d'amore; et rigittando la colpa in esso Amore, alle forze del quale dimostra non haver potuto far resistenza, iscusandosi per l'età. ${ }^{32}$

La critica più attenta al problema dell'esordio nelle raccolte liriche, si è sempre concentrata sulla parte centrale della nota, quella cioè che, in accordo con altri commenti cinquecenteschi, ritiene la cronologia della scrittura del sonetto in aperto contrasto con la sua collocazione nell'ordine dei componimenti. E invece passata sotto silenzio l'altra e successiva chiosa, quella che qualifica il sonetto "come Proemio fatto per insinuatione ». Con essa il Daniello si riferisce, com'era allora ovvio ed oggi evidente, alle trattazioni contenute, di nuovo, nel De inventione $e$ nella Rhetorica ad Herennium le quali definiscono con tale nome il genus d'esordio da utilizzarsi quando, scrive Cicerone, " animus auditoris infestus est ». Tre sono le causae per le quali si verifica questa evenienza:

aut cum turpem causam habemus, hoc est cum ipsa res animum auditoris a nobis alienat; aut cum animus auditoris persuasus esse videtur ab iis, qui ante contra dixerunt; aut cum defessus est eos audiendo, qui ante dixerunt. ${ }^{33}$

Di tali tre circostanze, quella a cui il Daniello fa riferimento è senz̧a dubbio la prima: Petrarca scrive il suo sonetto proemiale "preoccupandosi in esso quello c'baverbbon potuto dir gli ascoltanti », a motivo della discrepanza fra la gravità della sua persona e la modestia della materia trattata (l'amore rientra dunque, sarà bene ribadirlo, nel genus humile). Se si riconduce il tutto agli schemi dei manuali retorici, si deve concludere, con la pedanteria e la diligenza 
del commentatore lucchese, che il problema di Petrarca è dunque la sua stessa causa, il genus turpe cui essa appartiene. Ė questa infatti che gli fa mancare la buona disposizione all'ascolto e gli impedisce di ricercare apertamente l'attenzione, la docilità, la benevolenz̧a dell'uditorio, obbligandolo dunque a farlo in modo coperto, insinuante appunto. Percio, sostiene il Daniello, il poeta distrae da sé l'attenzione dei giudici, "rigittando» la colpa, poiché di colpa certo si tratta, su un'altra persona, quella d'Amore, e su un altro tempo, quello dell' età giovanile.

La chiave di lettura proposta dallo scolaro di Trifon Gabriele porta dunque l'analisi del testo sul terreno dell'arte oratoria e se si continua ad adoprare lo strumento ch'egli ci indica, il testo petrarchesco rivela la presenza di un'articolazione retorica che permette di leggere in modo diverso da quello sinora tenuto anche taluni elementi stilistico-formali.

Assai utile risulta, a questo proposito, lo studio del sonetto incipitario del Canzoniere svolto da Noyer-Weidner il quale, fra le molte altre cose e all'interno d'un ragionamento di più ampia portata, dedicò particolare attenzione proprio alla sintassi del testo, individuandovi una traccia rivelatrice della poetica petrarchesca e delle sue tensioni. ${ }^{34}$ In estrema sintesi: nella sua lettura il critico tedesco riconduceva anche questo tratto formale alla duplice matrice della cultura del poeta e cosi la diversità ch'egli riscontrava fra le quartine e le terzine era fatta dipendere da categorie stilistico-ideologiche secondo le quali le prime sarebbero state ciceroniane e classiche, le seconde cristiane e medievali. Seguiamo però con un po' più di cura l'argomentazione di Noyer-Weidner. Nel comporre il primo sonetto del Canzoniere Petrarca riprende, nella fronte, elementi propri della lirica stilnovistica ma

il materiale ereditato dalla tradizione viene da Petrarca fortemente modificato [...]; quanto al contenuto, per mezzo di tratti volutamente soggettivanti; quanto alla forma, sia sul piano metrico che su quello linguistico, e su quest'ultimo dalla semantica lessicale fino alla sintassi, nella direzione di un insieme consciamente intricato. ${ }^{35}$

Come già anticipato il giudizio di Noyer-Weidner è chiaro: «la costruzione sintattica della fronte appare quasi 'ciceroniana' $»,{ }^{36}$ ed è perciò "connessa, nel senso classico di poetica e retorica, con un'esigenza artistica elevata $\gg,{ }^{37}$ segno questo dell'aspirazione petrarchesca alla gloria e al superamento della precedente tradizione lirica. Tutt altra invece la struttura della sirma: essa è "trasparente » e presenta "una sintassi relativamente semplice ». ${ }^{38}$ Proprio questa semplicità - unita alle vistose figure di suono di quei versi e alle affinità stilistiche e di contenuto col Secretum - indica l'adesione a un "sermo bumilior», ${ }^{39}$ opposto a quello classicheggiante delle quartine, che rimanda al sistema della retorica cristiana e che viene qui usato "a significare la 
superiorità spirituale rispetto a tutto ciò che è profano, compreso lo stile elevato classico $\gg{ }^{40}$

Anche Noyer-Weidner, analizzando la sintassi e lo stile, giunge dunque, come il Daniello, a parlare di retorica: nel suo caso, di differenti sistemi retorici e, più in là, di differenti modelli culturali. Contrariamente però a quanto egli sosteneva, a me pare che le macroscopiche differenze sintattiche e d'andamento delle due parti del sonetto siano da ricondurre non tanto a categorie ideologiche, di necessità un po' schematiche, quanto piuttosto a un'unica e precisa strategia retorica, tutta quanta interna al testo: insinuatio scrive il Daniello, e dice bene. Interessante a questo proposito una notazione dello stesso NoyerWeidner: " non è in forma peculiarmente proemiale, ma un po' in sordina che Voi ch'ascoltate introduce - secondo contenuto, 'stile' e significato - le poesie che seguono $\gg{ }^{4 \mathrm{I}}$

Il sonetto, lo si è visto, procede da un massimo a un minimo di complicazione sintattica e, specularmente, da un minimo ad un massimo di trasparenza argomentativa: quanto sono intricate le quartine, tanto sono semplici le terzine. Quasi insomma, aggiungo, che l'eterno dilemma petrarchesco di un mutamento imperfetto, di un 'già ma non del tutto', fosse espresso proprio da quel viluppo di complicazione sintattica e ambiguità semantica ${ }^{42}$ contenute nella fronte, complicazione e ambiguità che fanno smarrire la chiarezza dei nessi di causa-effetto e il discernimento fra ciò che è vero e ciò che è falso. All'opposto, nella sirma, si distendono invece due periodi la cui linearità e perspicuità rispecchia perfettamente le constatazioni che l'uso retto della ragione mostra come inevitabili all'uomo. Cosi, nella prima parte, l'argomentazione di Petrarca fa di tutto per dire la colpa dell'nomo che Petrarca fu e, al tempo stesso, per nasconderla, per farla scivolare in un secondo piano grazie a una capziosa inversione dei rapporti logici fra le frasi, per mezzo di una voluta complicazione delle relazioni di causa-effetto, la quale realizza, in definitiva, una sorta di cortina di fumo, dalla quale l'ascoltatore riemerge un po' frastornato (anche per l'ambiguità di certe espressioni petrarchesche, cosi sfumate da risultare quasi generiche) e avendo presente ormai una sola cosa, ovvero quel che Petrarca chiede: "pietà nonché perdono ». Giunti a questa richiesta, che sola interessa il poeta, patronus di se stesso, giunti alla descrizione dell'uomo di oggi che lucidamente comprende la condizione passata e la realtà vera delle cose, tutto si fa squadrato nella netta corrispondenza di sintassi e metro, e l'ultima immagine lasciata alla giuria - il «Voi ch'ascoltate » d'attacco, sospeso, genericamente allocutorio, privo di reggenza verbale - è quella di un penitente - il famoso ribattere della ' $m$ ' fa risuonare le parole autoaccusatorie del Confiteor e i colpi sul petto che le accompagnano - è l'effige di un nomo mutato e ravveduto, di un saggio capace di chiudere in una massima l'assenza di significato del comune esistere degli uomini. 
Se, tenendo a mente queste note, si torna a rileggere il paragrafo del De inventione che spiega come si costruisca un esordio per insinuazione, è difficile sottrarsi all'idea che l'eccezionali ambiguità e densità semantica del dettato petrarchesco altro non siano che la mirabile veste con la quale si ammanta il nudo elenco dei precetti ciceroniani:

Si causae turpitudo contrahit offensionem, aut pro eo homine, in quo offenditur, alium hominem, qui diligitur, interponi oportet; aut pro re, in qua offenditur, aliam rem, quae probatur [...] ut ab eo, quod odit, ad id, quod diligit, auditoris animus traducatur; et dissimulare te id defensurum, quod existimeris; deinde, cum iam mitior factus erit auditor, ingredi pedetemptim in defensionem et dicere ea, quae indignentur adversarii, tibi quoque indigna videri; deinde, cum lenieris eum, qui audiet, demonstrare, nihil eorum ad te pertinere et negare quicquam de adversariis esse dicturum, neque hoc neque illud, ut neque aperte laedas eos, qui diliguntur, et tamen id obscure faciens, quoad possis, alienes ab eis auditorum voluntatem; et aliquorum iudicium simili de re aut auctoritatem proferre imitatione dignam; deinde eandem aut consimilem aut maiorem aut minorem agi rem in praesenti demonstrare. ${ }^{43}$

Petrarca, per quel che di questo passo si attaglia al suo discorso, pare davvero seguire in tutta umiltà il suo maestro d'eloquenza: all'uomo di un tempo sostituisce, almeno «in parte », quello presente e al " primo giovenile errore » contrappone la "vergogna » che adesso gli segna il volto, in modo da far cadere lontano da sé, verso un altro e un altrove, quell" offensionem », che gli sottrae il favore dell'uditorio. Non solo: Petrarca sembra rispettare, oltre la sostanza dei precetti, anche la modalità d'esecuzione, quel « dissimulare » e quell" « ingredi pedetemptim " con cui dev'essere condotta la difesa. Si noti infatti come per tutta la fronte del sonetto egli elenchi i capi d'imputazione, le proprie colpe, prepari lo stacco del nono verso ma come, proprio con tale elenco e tale preparazione, finalizzate alla richiesta di "pietà, nonché perdono", egli dissimuli la difesa della propria opera, la possibilita cioè che essa sia accolta e ascoltata. Ancora: la supplica è preceduta dalla specificazione di un'eventualità - quella che all'interno di quel voi in ascolto ci sia « chi perprova intenda amore» - che invita $i$ giudici a specchiarsi nell'imputato, sollecitandone la mitezza e quasi ricordando loro che altri, per cause simili, erano stati gindicati con indulgenza. Ė solo a questo punto che, a correggere le residue riluttanze del " quand'era in parte », giunge il forte strappo del "Ma ben veggio or sì tramite il quale il poeta fa proprio il punto di vista del "popol tutto", mostrando che ora anch'egli biasima quel che gli altri biasimavano, e che le accuse che gli potrebbero essere mosse non banno alcuna attinenza con $i$ pensieri e la consapevolezza dell'uomo che egli è. E però anche a questo si giunge poco a poco, facendo cioè passare il rincrescimento per le proprie colpe da 
una determinazione temporale - " sovente / di me medesmo » - alla assolutezza della conoscenza condensata nel verso conclusivo. I due tempi del sonetto, il suadente legato della fronte e lo staccato vigoroso della sirma, trovano cosi una motivazione non soltanto nell' ideologia' e nella 'poetica' ma, e forse in modo più preciso, nelle sottigliezze e nelle ambiguità caratteristiche dell'insinuatio.

Il Bembo invece, lo si è visto, si comporta in modo affatto diverso; lo può fare perché diversi sono i generi di causae che tratta, perché nessuna di esse $\grave{e}$ del tutto disdicevole. Perciò quello del veneziano è un vero e proprio "principium », o, alla greca, "prohemium», laddove quello del Petrarca realizza la forma più difficile e, se si vuole, rara, quella dell" insinuatio »o "epodos» per $i$ retori greci. ${ }^{44}$ La questione non è meramente nominale e, soprattutto per il Bembo, non è certo trascurabile. Se pure infatti, per quanto riguarda Voi ch'ascoltate, si volesse attribuire il ricorso ai precetti retorici del De inventione o della Rhetorica ad Herennium alle inveterate abitudini critiche di un commentatore cinquecentesco e, più in generale, dei lettori intendenti del secolo, in Piansi et cantai la scelta non poté che essere fatta con piena consapevolezza poiché Bembo, pur avendo di fronte a sé l'esempio petrarchesco, lo ignorò deliberatamente, optando per il tipo d'inizio contrario.

Tale scelta dimostra che il modello del sonetto d'esordio del Canzoniere era tutt'altro che irrinunciabile, risultava anzi, per certi versi, desueto, persino sconveniente nella mancanza di decoro con la quale il poeta vi confessava apertamente, in pubblico, gli errori d'un'esistenza $a^{45}$ e non le carenze formali di un opera, come appunto avrebbe fatto il Bembo. La normalizzazione ch'egli attua, tante volte rilevata dai lettori e dai critici delle sue Rime in questi ultimi anni, passa attraverso uno spostamento che, prima ancora d'essere tematico, è retorico. Un 'vero' proemio, ben lo sapeva il Bembo, non poteva essere come quello del Petrarca. Era necessario anzi tutto fare chiarezza, districare l'ambiguo intreccio di esperienza esistenziale e poetica, facendo passare nettamente l'oggetto della causa dall'uomo all'opera, cambiando quindi il genere della causa, e cosi facendo, sgombrare il campo da ogni possibile autoaccusa, da ogni dichiarazione di pentimento e di conversione, dati incoerenti oramai con i riferimenti culturali d'un'età nuova e diversa. Quanto poi alla potenziale scabrosità della materia esposta nella prima quartina, essa era neutralizzata dapprima relegandola, senza mezzi termini, in un altrove temporale, in un passato senza più rapporti col presente, e poi racchiudendola nella duplice cornice della didassi e della pia sapienza, facendo cioè dello strazio amoroso un mezzo d'insegnamento, il gradus iniziale, per chi fosse lettore " accorto ", ${ }^{46}$ verso l'ascesa alle più alte sfere della contemplazione platonica e cristiana. 
In questo quadro, il ritorno a un uso meno sottile dei precetti retorici e il recupero d'una modalità d'esordio più evidentemente proemiale - l'invocazione alle Muse potrebbe essere considerata alla stregua d'un esempio d'ipercorrettismo - rappresentano la ripresa e la proposta ostentata, integrale, della norma con la conseguente emarginazione della variatio, anche quella, sempre esatta, ammiratissima, che si poteva ritrovare nel maestro riconosciuto, nel modello prescelto per la lirica volgare.

A questo già lungo ragionamento, mi sia consentito d'aggiungere una breve 'coda' che spero riesca a fornire una conferma indiretta a quanto fin qui sostenuto. Come si sa, Piansi et cantai "probabilmente venne composto intorno al $1529 »,{ }^{47}$ in previsione della stampa del I 530 : la rigidità con la quale è costruito e la muscolare consapevolezza teorica che lo contraddistingue volevano essere forse il primo atto d'una strategia letteraria tesa a mascherare il più possibile la lunga vicenda e le tensioni interne del canzoniere, ${ }^{48}$ a ricomporle in un edificio che fosse, fin dalla facciata, splendido e serenissimo. Per questo, forse, anche Bembo, cosi contrario a tenere sempre «la regola dell'arte con la penna in mano", nel proemio, un luogo tanto esposto, avrà sospeso l'adesione al proprio, nuovo, ideale di retorica « discorsivo e privato » per cedere a quello « oratorio e pubblico » dell' antichità. ${ }^{49}$ Una concessione che rappresentava il dazio pagato all'esplicita volontà di porsi come modello.

Una vicenda lunga, s'è detto, quella del canzoniere del Bembo, fors'anche troppo: negli anni '30, quand'essa giunge finalmente al termine e la stampa consacra l'esemplarità di quell'esercizio poetico, pei territori della lirica volgare, già si cercano altre vie, diverse, divergenti talora da quelle segnate dal veneziano. L'anno seguente, nel IS3I, giungeva ai torchi veneziani dei Nicolini da Sabbio il Libro primo degli Amori di Bernardo Tasso: come si sa la lettera di dedica premessa all'opera è una sorta di 'volantino rivoluzionario' che invita all'imitazione degli antichi, abbandonando quella dei moderni. È notevole che in questione siano anche le modalità compositive del testo proemiale delle raccolte liriche: dice infatti il Tasso che, alla fine del suo libro, ha aggiunto

alcune altre poche rime, cantate secondo la via e l'arte degli antiqui boni poeti greci e latini, i quali sciolti d'ogni obligazione, cominciavano e fornivano i loro poemi com' a ciascun parea [...] né aveano rispetto di principiar più con proemio che senza, o se pure il facevano, non curavano di dargli quelle parti che quel della prosa ricerca $\left[\ldots . . .^{5 \circ}\right.$

L'analisi qui condotta, ricorrendo ai manuali d'oratoria, sul sonetto incipitario delle Rime bembiane dovrebbe aver chiarito, fin troppo, a cosa faccia riferimento il Tasso parlando d'un proemio che abbia le "parti [...] della prosa». Nell'opposizione tassiana alla norma appena instaurata, si può 
cogliere poi un segno di novità nel modo in cui essa viene condotta, perché se il disaccordo del Bembo nei confronti dell'inizio petrarchesco era restato tutto implicito nella forma di cui Piansi et cantai faceva mostra, il Tasso non si contentava solo d'un'opposizione in re - attuata d'altra parte col sonetto iniziale del Libro primo degli Amori - ma, a chiare lettere, portava la polemica sul piano della teoria e della storia letteraria. Egli non nomina il Bembo, anzi; tuttavia fra le raccolte d'autori importanti uscite in quel giro d'anni, solo le Rime del Is30 presentano, se non m'inganno, un prohemium che osserva tutte le "parti » prescritte ed adempie a tutti gli officia previsti. Per questo l'obiettivo polemico del Tasso sembra essere proprio Piansi et cantai, e l'impazienza nei confronti di norme e precetti che s'avverte nelle sue parole pare davvero essere la reazione uguale e contraria alla diligente osservanza alle regole dell'arte con la quale il Bembo ci è sembrato avesse voluto costruire il suo testo.

Giovanni Ferroni 


\section{Giovanni FERroni}

Si presenta qui, con significative modifiche e ampliamenti, la comunicazione letta in una delle sessioni parallele del Convegno dell'ADI (Roma, I7-20/9/2008) e il cui testo sarà pubblicato negli atti.

I. Il riferimento è al lungo articolo di A. Noyer-Weidner, Lyrische Grundform und epischdidaktischer Überbietungsanspruch in Bembos Einleitungsgedicbte, in « Romanische Forschungen », LXXXVI (1974), pp. 314-58.

2. Cfr. N. Gardini, Bembo: un codice volgare per la lirica moderna, in Id., Le umane parole. L'imitazione nella lirica europea del Rinascimento da Bembo a Ben Jonson, Milano, Bruno Mondadori, 1997, in particolare le pp. 67-73. Si veda però anche D. Della Terza, Imitatio: Theory and Practice. The example of Pietro Bembo, in "Yearbook of Italian Studies", I97 I, pp. I I9-4I (si cita però dalla trad. it. Imitatio: teoria e pratica. L'esempio del Bembo poeta, in Id., Forma e memoria, Roma, Bulzoni, 1979, pp. I I 5-47).

3. Si vedano L. Russo, Pietro Bembo e la sua fortuna storica, in «Belfagor», XIII (1958), pp. 257-72, ma anche Noyer-Weidner, Lyrische Grundform, cit., pp. 314-23.

4. Si cita da Pietro Bembo, Le Rime, a cura di A. Donnini, Roma, Salerno, 2008, i, pp. 6-8.

5. Russo, Pietro Bembo, cit., pp. 269-70; Noyer-Weidner, Lyrische Grundform, cit., n. 50, p. 342.

6. Francesco Petrarca, Triumphus Cupidinis, ilI, i 42 (si cita dall'edizione curata da M. Ariani, Milano, Mursia, 1988).

7. Si cita la lezione della stampa i 530 secondo l'edizione contenuta nei Poeti del Cinquecento, a cura di G. Gorni, M. Danzi, S. Longhi, Milano-Napoli, Ricciardi, 200I, pp. 5 I- 22.

8. Si fa qui riferimento alla Rhetorica ad Herennium perché era, nel Rinascimento, fra i più noti manuali di retorica antica e perché presenta, con quella ciceroniana del De inventione, la più ampia e sistematica trattazione sull'exordium, trattazione per lo più coincidente a causa, com'è noto, della dipendenza da una comune fonte ermagorea. Citerò per questo da entrambi i testi, usando ora l'uno ora l'altro a seconda del caso. Per un quadro complessivo delle dottrine antiche su questa parte del discorso si veda L. Calboli Montefusco, Exordium narratio epilogus: studi sulla teoria retorica greca e romana delle parti del discorso, Bologna, CLUEB, I988, pp. I-32. Sulla Rhetorica ad Herennium si veda l'Introduzione premessa a CorNIFICI, Rhetorica ad C. Herennium, introduzione, testo critico, commento a cura di G. Calboli, Bologna, Pàtron, $1993^{2}$, edizione da cui si cita e dalla quale si accoglie, senza entrare nel merito, l'attribuzione cornificiana - per altro corrente già nel Cinquecento: in questa sede, il problema della paternità dell'opera può infatti essere tralasciato senza danno. Per il $D e$ inventione si fa riferimento a M.T. CiCERONE, De inventione, introduzione, traduzione e note a cura di M. Greco, Galatina, Congedo Editore, 1998.

9. Cornifici, Rhetorica ad C. Herennium, I, III, 4, p. 96.

Io. Cornifici, Rhetorica ad C. Herennium, I, Iv, 7, p. 98.

i i. Giuseppe Baretti, La frusta letteraria, xxv, in ID., Opere scelte, a cura di B. Maier, Torino, UTET, r 972, pp. 586-605: p. 600.

I2. Cornifici, Rhetorica ad C. Herennium, I, Iv, 7, p. 98.

I 3. Ricordo che su questo punto vi è una lieve discrasia, non rilevante ai nostri fini, tra i due manuali di retorica che qui si tengono presenti: mentre nella Rhetorica ad C. Herennium (I, III, 5; ed. cit. p. 97) sono menzionati quattro « genera causarum [...]: honestum, turpe, dubium, 
humile », nel De inventione (I, xv, 20; ed. cit. p. 96) essi sono cinque: « honestum, admirabile, humile, anceps, obscurum ». Sulla questione si vedano L. Calboli Montefusco, Exordium narratio epilogus, cit., pp. 8-1o e CoRNIFICI, Rhetorica ad C. Herennium, n. I 3, pp. 2 I I-I 2.

I4. Cicerone, De inventione, I, xv, 20, pp. 96-98.

i s. BAREtTi, La frusta letteraria, p. 6o I.

16. Noyer-Weidner, Lyrische Grundform, cit., pp. 332 sgg.

17. Torquato Tasso, Discorsi del Poema eroico, in Id., Discorsi dell'Arte poetica e del Poema eroico, a cura di L. Poma, Bari, Laterza, 1964, pp. 174-75.

I 8. F. Erspamer, Il canzoniere rinascimentale come testo o come macrotesto: il sonetto proemiale, in «Schifanoia », IV (1987), 4, pp. I09-1 4: p. I I 2.

19. Cfr. il cappello introduttivo di Donnini a Piansi et cantai in Bembo, Le Rime, p. 5.

2o. Ivi, p. 7 .

2 r. Ovidio, Opere, a cura di P. Fedeli, vol. I, Torino, Einaudi, 1999, p. 52.

22. Gli altri tre per la Rhetorica ad C. Herennium, come anche per il De inventione, sono «ab nostra, ab adversariorum nostrorum [...] persona, et a rebus ipsis » (I, v, 8, p. 98).

23. Cicerone, De inventione, I, xvi, 22, p. ioo.

24. Si veda nella Rhetorica ad C. Herennium (I, v, 8, p. 98) il passo parallelo a quello citato che suona: «si res eorum fortiter, sapienter, mansuete, magnifice iudicatas proferemus; et si, quae de iis existimatio, quae iudicii expectatio sit, aperiemus ».

25. Per la diversità dei nomi dei genera cfr. qui la n. I 3 .

26. Cicerone, De inventione, I, xv, 2 I, p. 98.

27. Cornifici, Rhetorica ad C. Herennium, I, v, 8, p. $9^{8}$.

28. Cornifici, Rhetorica ad C. Herennium, I, Iv, 7, p. $9^{8}$.

29. Il rapporto infatti che intercorre fra le parti del sonetto del Bembo e i passi della Rhetorica ad Herennium e del De inventione dev'essere considerato in modo non eccessivamente stringente $\mathrm{e}$, comunque, in modo non più stretto di quanto si sia già fatto qui. Relativamente all'uso degli strumenti della retorica, non si deve infatti dimenticare la restrizione al concetto di 'persuasione' che il Bembo opera nel capitolo xIx del secondo libro delle Prose della volgar lingua (Pietro Bembo, Prose e rime, a cura di C. Dionisotti, Torino, UTET, 1966², pp. 173-74): le opere degli scrittori risultano inefficaci «se ancora questa rapitrice degli animi di chi ascolta esse non hanno dal lor canto. La quale a dissegnarvi e a dimostrarvi bene e compiutamente, quale e chente ella è, bisognerebbe tutte quelle cose raccogliere che dell'arte dell'orare si scrivono [...]. Ma io non dico ora persuasione in generale e in universo; ma dico quella occulta virtù, che, in ogni voce dimorando, commuove altrui ad assentire a ciò che egli legge, procacciata più tosto dal giudicio dello scrittore che dall'artificio de' maestri. Con ciò sia cosa che non sempre ha, colui che scrive, la regola dell'arte con la penna in mano. » Per l'importanza di questo passo nell'ottica del commento e dell'imitazione di Petrarca si veda P. Zaja, Per una sistemazione retorica del commento al Canzoniere: appunti intorno alle postille inedite di Giulio Camillo, in Il commento e i suoi dintorni, a 


\section{Giovanni Ferroni}

cura di B.M. Da Rif, Milano, Guerini e associati, 2002, pp. I05-28.

30. La formula è tratta da Gardini, Bembo: un codice volgare per la lirica moderna, cit., p. 67.

31. A proposito di questo commento il rimando, obbligato, è allo studio di E. Raimondi, Bernardino Daniello lettore di poesia, in Id., Rinascimento inquieto, Torino, Einaudi, I994, pp. 19-56. Si veda anche G. Belloni, Bernardino Daniello e le varianti d'autore, in ID., Laura tra Petrarca e Bembo. Studi sul commento umanistico-rinascimentale al Canzoniere, Padova, Editrice Antenore, 1992, pp. 226-83.

32. Sonetti, canzoni, e triomphi di messer Francesco Petrarcha con la sposizione di Bernardino Daniello da Lucca, in Vinegia per Giovanni Antonio de Nicolini da Sabio, MDxul, c. A r $r$.

33. Cornifici, Rhetorica ad C. Herennium, I, vI, 9, pp. 98-99.

34. A. Noyer-Weidner, Il sonetto I, in Lectura Petrarce, IV, I984, Firenze, L.S. Olschki, I 985 , pp. 327-53 (si vedano in particolare le pp. 333-42).

35. Noyer-Weidner, Il sonetto I, cit., p. 34I.

36. Ibidem.

37. Noyer-Weidner, Il sonetto I, cit., p. 342.

38. Noyer-Weidner, Il sonetto I, cit., p. 343-44.

39. Noyer-Weidner, Il sonetto I, cit., p. 347.

40. Noyer-Weidner, Il sonetto I, cit., p. 348 .

4I. Noyer-Weidner, Il sonetto I, cit., p. 329.

42. Sono osservazioni che Noyer-Weidner presenta e precisa in più punti del suo studio: «la sintassi» scrive «non solo è complicata ma tende all'ambivalenza, e Petrarca se ne serve volutamente, [...] in funzione di un gioco combinato di correlazioni molteplici o per lo meno varie possibilità di riferimenti »; per quanto poi riguarda la semantica egli parla, più in generale, di una «tendenza tipicamente petrarchesca verso l'ambivalenza o piuttosto la plurivalenza di significato e di funzione dell'enunciato » (ivi, p. 338-39; p. 35 I).

43. Cicerone, De inventione, I, XVII, 24, p. IO2.

44. I termini latini e greci con i quali si indicano i generi di esordio li cito da CoRNIFICI, Rhetorica ad C. Herennium, I, IV, 6, pp. 97. Circa la differenza fra principium e insinuatio così si esprime la Rhetorica ad Herennium: «Inter insinuationem et principium hoc interest. Principium eius modi debet esse, ut statim apertis rationibus, quibus praescripsimus, aut benivolum, aut attentum, aut docilem faciamus auditorem: at insinuatio eiusmodi debet esse, ut occulte per dissimulationem eadem illa omnia conficiamus, ut ad eandem commoditatem in dicendi opera venire possimus » (ivi, I, vII, I I, p. 99).

45. Di questo disagio nei confronti di Voi ch'ascoltate è testimonianza eloquente quel che proprio il Bembo scriveva all'Astemio il 4 di luglio I 5 29: "Onorato M. Nicolò Dio vi salvi. Se il Petrarca non v'ha potuto persuadere egli d'essere stato veramente innamorato di Mad. Laura, con tanti suoi belli e cari scritti volgari, e spezialmente col primo suo sonetto nel quale non è verisimile che egli fingesse a sua vergogna, e con tanti altri latini ne' quali egli fa testimonio di ciò, io non presumerò già di poterlovi persuadere io. » (in Pietro Bembo, 
Lettere, vol. III, ed. critica a cura di E. Travi, Bologna, Commissione per i testi di lingua, I 992, 997, pp. 65-66). Altri giudizi del Bembo su Petrarca e suoi interventi su singoli fragmenta sono raccolti da E. Travi, Come Pietro Bembo leggeva Petrarca, in «Testo », 27 (I 994), pp. 49-55.

46. La iunctura « amanti accorti » non è petrarchesca (lo segnala D. Della Terza, Imitatio: teoria e pratica. L'esempio del Bembo poeta, cit., p. I43); io l'intendo non tanto, come mi sembra fare Donnini nel suo commento, come 'cauti' nei confronti dei danni possibili causati dall'amore, ma nel senso di 'giudiziosi, intelligenti' capaci cioè di leggere in modo acuto la poesia. Concordo quindi con l'interpretazione data da Ugolino Martelli (il cui testo però leggeva 'genti accorte') nella Lezione su Piansi e cantai pronunciata il i 9 di settembre i 540 e dunque vivente il Bembo - all'Accademia degli Infiammati di Padova: «Le genti accorte: non dico le genti rustiche e grossolane ma le sagge e avvedute. Stimino pure quanto si vogliono gli sciocchi e ignoranti uomini che tutti i poeti siano baie e fole di romanzi, e tenghino per fermo i saggi e prudenti che non punto manco e di dottrina e di boni costumi si può cavare de i buoni e approvati poeti, che si cavi delle scuole e de i libri de i filosofi, pure che da persone accorte e giudiziose siano letti e considerati ». Ringrazio moltissimo Franco Tomasi che mi ha segnalato la Lezione del Martelli e che mi ha permesso di vedere la sua trascrizione del manoscritto conservato alla Biblioteca Civica di Padova (segnatura B.P. I 830 ; il passo citato si trova alla c. I I $v$ ); per una descrizione del testimone (e la relativa bibliografia) si veda la scheda curata da Gino Belloni in Petrarca e il suo tempo, Milano, Skira, 2006, pp. 527-28. L'itinerario, non solo biografico, del Martelli è stato ricostruito da V. Bramanti, Ritratto di Ugolino Martelli (III9-I592), in "Schede umanistiche » n.s., 2 (I 999), pp. 5-53; chiarisce invece gli orientamenti culturali e la prassi esegetica che caratterizzavano l'accademia padovana F. Tomasi, Le letture di poesia e il petrarchismo nell'Accademia degli Infiammati, in Il Petrarchismo. Un modello di poesia per l'Europa, II, a cura di F. Calitti e R. Gigliucci, Roma, Bulzoni Editore, 2006, pp. 229-50.

47. Bемво, Le Rime, p. 5 .

48. Cfr. il profilo tracciato da Gorni nel cappello introduttivo alla sezione dedicata a Bembo dei Poeti del Cinquecento, cit. (in particolare le pp. 42-43).

49. Si veda il passo delle Prose della volgar lingua qui citato alla n. 29 e la corrispondente chiosa di Dionisotti.

50. Bernardo Tasso, Rime, vol. i, a cura di D. Chiodo, Torino, Res, I995, p. I6. 